\title{
Persistem iniquidades sociais na distribuição da cárie dentária em adolescentes maranhenses? Contribuições de um estudo de base populacional
}

\author{
Do social inequalities persist in the distribution of dental caries \\ in adolescents from Maranhão? Contributions \\ of a population-based study
}

\author{
Francenilde Silva de Sousa (http://orcid.org/0000-0003-0681-7620) ${ }^{1}$ \\ Brenda Costa Lopes (http://orcid.org/0000-0003-2949-2961) ${ }^{2}$ \\ Elisa Miranda Costa (http://orcid.org/0000-0001-5364-0384) ${ }^{1}$ \\ Cláudia Maria Coelho Alves (http://orcid.org/0000-0003-4705-4914) ${ }^{2}$ \\ Rejane Christine de Sousa Queiroz (http://orcid.org/0000-0003-4019-2011) ${ }^{1}$ \\ Aline Sampieri Tonello (http://orcid.org/0000-0002-8402-9112) ${ }^{1}$ \\ Cecília Cláudia Costa Ribeiro (http://orcid.org/0000-0003-0041-7618) ${ }^{2}$ \\ Erika Barbara Abreu Fonseca Thomaz (http://orcid.org/0000-0003-4156-4067) ${ }^{1}$
}

${ }^{1}$ Departamento de Saúde Pública, Universidade Federal do Maranhão (UFMA). R. Barão de Itapari 155, Centro. 65020 070 São Luís MA Brasil. fraansoousa@gmail.com ${ }^{2}$ Departamento de Odontologia, UFMA. São Luís MA Brasil.

\begin{abstract}
This study aimed to analyze the association of socioeconomic factors with the prevalence of dental caries in adolescents from São Luís, Maranhão, Brazil, to answer whether social inequalities persist in distributing this disease. This is a cross-sectional study nested in a prospective cohort. We included 2,413 adolescents aged 18-19 years evaluated in the 2016 second follow-up. The outcome was teeth with untreated dental caries (yes or no) assessed by the DMFT index. Socioeconomic and demographic characteristics were the independent variables. Descriptive statistical and Poisson regression analyses were performed, calculating crude and adjusted prevalence ratios (PRs) (alpha=5\%). Belonging to economic classes C (PR=1.23; 95\% CI: 1.11-1.37) or D-E ( $P R=1.48 ; 95 \%$ CI: 1.32-1.65), being married/ living with a partner $(P R=1.22$; $95 \%$ CI: 1.07 1.39), having separated parents ( $P R=1.11 ; 95 \%$ CI 1.03-1.19) and a greater number of people in the household ( $P R=1.05$; 95\% CI: 1.03-1.07) were associated with a higher prevalence of dental caries. Social inequalities in adolescent oral health persist despite the implementation of the National Oral Health Policy. The current health care model should seek to reorient health education strategies, targeting them at vulnerable populations.

Key words Dental caries, Socioeconomic factors, Adolescent
\end{abstract}

Resumo O objetivo foi analisar a associação de fatores socioeconômicos com a prevalência de cárie dentária em adolescentes de São Luís, Maranhão, para responder se as iniquidades sociais persistem na distribuição desta doença. Este é um estudo transversal aninhado a uma coorte prospectiva. Incluímos 2.413 adolescentes de 18-19 anos, avaliados em 2016 ( $2^{\circ}$ seguimento). O desfecho foi a ocorrência de dentes com cárie dentária não tratada (sim ou não), avaliada pelo indice CPO-D. Características socioeconômicas e demográficas foram as variáveis independentes. Foram realizadas análises estatísticas descritivas e de regressão de Poisson, calculando-se razões de prevalência (RPs) brutas e ajustadas (alpha=5\%). Pertencer às classes econômicas $C(R P=1,23$; IC95\%:1,11$1,37)$ ou $D-E(R P=1,48$; IC95\%: 1,32-1,65), estar casado/morar com companheiro $(R P=1,22$; IC95\%:1,07-1,39), ter pais separados ( $R P=1,11$; IC95\%1,03-1,19) e maior número de pessoas na residência ( $R P=1,05$; IC95\%:1,03-1,07) foram associadas a maior prevalência de cárie dentária. Apesar da implementação da Política Nacional de Saúde Bucal, as iniquidades sociais em saúde bucal de adolescentes persistem. É fundamental que o modelo de atenção à saúde vigente busque a reorientação das estratégias de educação em saúde, direcionando-as a populações vulneráveis.

Palavras-chave Cárie dentária, Fatores socioeconômicos, Adolescente 


\section{Introdução}

Por sua elevada frequência, impactos econômicos e na qualidade de vida das pessoas ${ }^{1-3}$, a cárie dentária é apontada como um importante problema de saúde pública no Brasil ${ }^{4}$. É uma doença crônica resultante da dissolução mineral dos tecidos dentários proveniente da produção de ácidos por bactérias quando estas metabolizam carboidratos, em especial a sacarose, oriundos da dieta ${ }^{5}$.

Apesar de a etiologia da cárie ser bem conhecida, muitos aspectos relativos ao papel dos fatores socioeconômicos no processo vêm ganhando relevância e sendo abordados em vários estudos em associação aos determinantes biológicos que interagem na etiologia da doença ${ }^{6-8}$. Entretanto, alguns aspectos relativos ao papel desses fatores permanecem mal explicados.

A associação entre pobreza e desigualdades sociais com morbidades bucais tem sido objeto de estudos brasileiros ${ }^{9-11}$ e internacionais ${ }^{6,12,13}$ e são levantadas diferentes explicações teóricas para a causação social, a exemplo da teoria da qualidade social ${ }^{1,2}$, teoria do curso da $v^{1} \mathrm{da}^{14}$, do estresse $^{15} \mathrm{e}$ do suporte social ${ }^{16}$. Existe a tese, em inúmeros estudos, de que a desvantagem socioeconômica está associada a maior incidência e prevalência de cárie, o que se justifica por maus hábitos comportamentais, maior vulnerabilidade aos fatores de risco para a doença e menor acesso ao tratamento ${ }^{1,6,17}$.

Entretanto, são raros os estudos de base populacional e com adequado controle de fatores de confusão desenhados para este fim, além de não haver consenso sobre exatamente quais fatores socioeconômicos têm papel mais relevante na ocorrência da doença. Ademais, com a ampliação da cobertura da Atenção Primária à Saúde (APS), voltada para a promoção da saúde, prevenção de doenças e tratamento das condições mais prevalentes, as evoluções nos modelos assistenciais e o crescimento da atenção à saúde bucal na rede pública, esperava-se uma redução nas iniquidades sociais ${ }^{18,19}$.

Visando contribuir para a qualidade das políticas de saúde bucal, o objetivo deste estudo foi identificar a prevalência de cárie dentária em adolescentes de São Luís, acompanhados na coorte RPS (Ribeirão Preto, Pelotas e São Luís Brazilian Cohort), e avaliar a sua relação com diferentes fatores socioeconômicos, ponderando se persistem iniquidades sociais na distribuição da cárie dentária.

\section{Métodos}

Trata-se de um estudo transversal aninhado a uma coorte prospectiva de nascidos vivos realizada no Município de São Luís-MA, Brasil. A coorte de origem é denominada RPS (por ter sido desenvolvida em Ribeirão Preto, Pelotas e São Luís). O estudo ocorreu de março de 1997 a fevereiro de 1998 (por ocasião do nascimento das crianças), baseline. As crianças foram reavaliadas em 2005 (idade escolar: entre 7-9 anos), considerado primeiro seguimento; e novamente em 2016, quando se encontravam na adolescência (18-19 anos de idade), segundo seguimento ${ }^{20}$.

A linha de base da coorte de nascimento incluiu recém-nascidos vivos de parto hospitalar de mães residentes no município de São Luís, de março de 1997 a fevereiro de 1998, e foi conduzida em dez hospitais públicos e privados. Utilizou-se amostragem sistemática com estratificação proporcional ao número de nascimentos em cada hospital. Assim, foram recrutados um em cada sete partos por hospital. Nessa fase, participaram 2.542 nascidos vivos. Após exclusão de natimortos, a amostra totalizou em 2.443 nascimentos $^{20}$.

No primeiro seguimento, a partir de censo escolar, todos os pais ou responsáveis pelas crianças localizadas que haviam nascido com baixo ou alto peso e um terço das demais foram convidados para uma reavaliação, totalizando 1.108 participantes elegíveis.

Aos 18 e 19 anos, os participantes dessa coorte passaram por nova avaliação, de janeiro a dezembro de 2016, o segundo seguimento. Para localizá-los, buscamos matrículas em escolas e universidades, endereços e contatos nas redes sociais, bem como registros de matrículas militares (para homens). No total, 654 adolescentes foram identificados e aceitaram participar dessa etapa. Devido à dificuldade de localização dos indivíduos e para ampliar o tamanho da amostra do estudo, incluímos os participantes de duas formas: por sorteio no banco de dados do Sistema de Informação sobre Nascidos Vivos (Cadastro Nacional de Nascidos Vivos - SINASC) ( $\mathrm{n}=1.716)$, e pela inclusão de voluntários identificados em escolas, universidades e redes sociais nascidos em maternidades de São Luís em 1997 (n=145). Esses novos participantes foram submetidos aos mesmos testes e questionários da coorte original ${ }^{21}$.

Assim, essa fase contemplou a participação de 2.515 adolescentes, porém, 102 destes foram retirados por estarem utilizando aparelho ortodôntico ou por recusa. Para este estudo, então, 
foram considerados dados de 2.413 participantes (Figura 1).

A coleta de dados foi realizada por profissionais da saúde treinados. Os dados referentes às características sociodemográficas, hábitos de vida e de consumo alimentar foram obtidos por questionários padronizados. As informações foram registradas no programa on-line Research Eletronic Data Capture ${ }^{22}$.

Estima-se que este tamanho de amostra tenha poder de $90 \%$ para identificar riscos relativos a partir de 1,5, considerando incidência de $50 \%$ de doença entre os expostos, razão de 1:1 entre expostos e não expostos, nível de confiança de $95 \%$ e efeito do desenho igual a 2,0.

As variáveis independentes foram: idade do adolescente (em anos); situação escolar do adolescente (estudando atualmente, sim ou não); história de trabalho atual do adolescente (sim ou não); situação conjugal do adolescente (solteiro, casado ou morando com companheiro); número

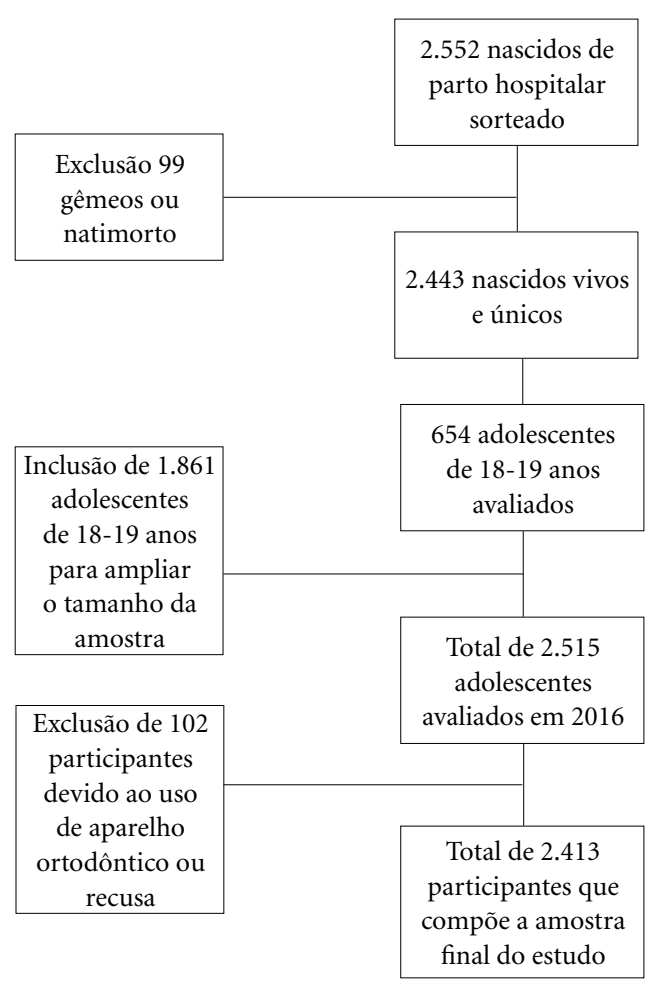

Figura 1. Fluxograma amostral do estudo. São LuísMA, Brasil, 1997-2016.

Fonte: Elaborado pelas autoras. de pessoas na residência; situação conjugal dos pais (separados ou não); renda familiar; o indicador de renda Poverty Income Ratio (PIR) ${ }^{23}$, que é a relação entre a renda familiar dividida pelo número de pessoas na residência, dividido por R\$ 140,00 (valor referente ao critério de pobreza, segundo o Banco Mundial e Governo Federal, 2016); benefícios sociais recebidos (sim ou não); quais benefícios sociais recebidos (aposentadoria, LOAS, Bolsa Família, pensão por morte ou doença); e classe econômica segundo os critérios da Associação Brasileira de Empresas de Pesquisa $(\mathrm{ABEP})^{24,25}$, distribuindo-os entre as classes $\mathrm{A}-\mathrm{B}$, C e D-E.

O desfecho foi a ocorrência de dentes com cárie não tratada (sim ou não), conforme o índice CPO-D modificado pela Organização Mundial da Saúde ${ }^{26}$. Esse dado foi coletado por meio de exame clínico odontológico, em consultório portátil, sob iluminação artificial, utilizando sonda milimetrada de ponta arredondada $n^{\circ} 11,5$ indicada pela Organização Mundial de Saúde ${ }^{26}$, no segundo seguimento.

Foram efetuadas análises estatísticas descritivas, testes bivariados e análises de regressão de Poisson, calculando-se razões de prevalência (RPs) brutas e ajustadas. Utilizou-se o software STATA versão 14 (Stata Corp., College Station, TX, USA), considerando alpha de 5\% para rejeição de H0.

O projeto foi aprovado no Comitê de Ética em Pesquisa (CEP) do HU-UFMA. O consentimento livre, após informação, foi concedido por escrito.

\section{Resultados}

A Tabela 1 resume as características socioeconômicas dos adolescentes incluídos no estudo, totalizando 2.413 participantes. Alguns indicadores chamam a atenção, a exemplo: $30,42 \%(n=734)$ não estavam frequentando a escola; $48,45 \%$ ( $\mathrm{n}=1.169)$ possuíam pais separados. A média da renda familiar foi de $\mathrm{R} \$ 2.421,03( \pm 5.132,72)$; $42,24 \%(\mathrm{n}=1.007)$ das famílias recebiam algum tipo de benefício social, como Bolsa Família, aposentadoria ou pensão por morte/doença. Conforme critérios da $\mathrm{ABEP}^{24,25}$, observou-se que 50,95\% $(\mathrm{n}=1.229)$ dos adolescentes pertenciam à classe $\mathrm{C} ; 24,96 \%(\mathrm{n}=602)$ pertenciam à classe $\mathrm{D}-\mathrm{E}$ e, segundo o $\mathrm{PIR}^{23}, 24,09 \%(\mathrm{n}=581)$ pertenciam à classe A-B. Segundo o indicador, 44,03\% $(\mathrm{n}=1.062)$ estavam três vezes ou mais acima da linha de pobreza, tendo, portanto, uma renda 
Tabela 1. Características da amostra do estudo (n=2.413). São Luís-MA, Brasil, 2016.

\begin{tabular}{|c|c|c|c|c|}
\hline Variáveis & $\mathbf{n}$ & $\%$ & média & dp \\
\hline \multicolumn{5}{|l|}{ Estudando atualmente } \\
\hline Não & 734 & 30,42 & & \\
\hline Sim & 1.658 & 68,71 & & \\
\hline Não informado & 21 & 0,87 & & \\
\hline \multicolumn{5}{|l|}{ Trabalhando atualmente } \\
\hline Não & 562 & 23,29 & & \\
\hline Sim & 380 & 15,75 & & \\
\hline Não informado & 1,471 & 60,96 & & \\
\hline \multicolumn{5}{|l|}{ Situação conjugal do adolescente } \\
\hline Solteiro & 2.300 & 95,32 & & \\
\hline Casado & 30 & 1,24 & & \\
\hline Morando com companheiro & 61 & 2,53 & & \\
\hline Viúvo & 1 & 0,04 & & \\
\hline Não informado & 21 & 0,87 & & \\
\hline Número de pessoas na residência & & & 4,41 & 1,58 \\
\hline \multicolumn{5}{|l|}{ Os pais do adolescente estão separados } \\
\hline Não & 1.223 & 50,68 & & \\
\hline Sim & 1.169 & 48,45 & & \\
\hline Não informado & 21 & 0,87 & & \\
\hline Renda familiar & & & $2.421,03$ & $5.132,72$ \\
\hline \multicolumn{5}{|l|}{ Poverty income ratio (PIR $)^{1}$} \\
\hline Pobre $(\mathrm{PIR}<1,0)$ & 478 & 19,82 & & \\
\hline Quase pobre $(1,0 \leq \mathrm{PIR}<2,0)$ & 438 & 18,16 & & \\
\hline Renda mediana $(2,0 \leq \mathrm{PIR}<3,0)$ & 412 & 17,08 & & \\
\hline Renda alta $(\mathrm{PIR} \geq 3,0)$ & 1.062 & 44,03 & & \\
\hline Não informado & 22 & 0,91 & & \\
\hline \multicolumn{5}{|c|}{ Alguém da residência recebe benefício social? } \\
\hline Não & 1,368 & 57,38 & & \\
\hline Sim & 1.007 & 42,24 & & \\
\hline Não informado & 38 & 1,57 & & \\
\hline \multicolumn{5}{|l|}{ Tipo do benefício social recebido } \\
\hline Seguro-desemprego & 36 & 1,49 & & \\
\hline Aposentadoria & 394 & 16,33 & & \\
\hline LOAS & 10 & 0,41 & & \\
\hline Bolsa Família & 504 & 20,89 & & \\
\hline Pensão por morte ou doença & 81 & 3,36 & & \\
\hline \multicolumn{5}{|l|}{ Classe econômica (ABEP) } \\
\hline A-B & 581 & 24,09 & & \\
\hline $\mathrm{C}$ & 1.229 & 50,95 & & \\
\hline $\mathrm{D}-\mathrm{E}$ & 602 & 24,96 & & \\
\hline
\end{tabular}

n: frequência absoluta. \%: frequência percentual. dp: desvio padrão. ${ }^{1}$ Relação entre a renda familiar dividida pelo número de pessoas na residência, dividido por R\$ 140,00 (valor referente ao critério de pobreza, segundo o Banco Mundial e Governo Federal, 2016). Valores abaixo de 1,0 significam família abaixo da linha de pobreza.

Fonte: Elaborado pelas autoras.

considerada alta; $17,08 \%(\mathrm{n}=412)$ possuíam uma renda mediana; $18,16 \%(n=438)$ eram considerados pobres; e 19,82\% ( $n=478)$ estavam abaixo do critério de pobreza.

Cinquenta e seis por cento $(n=1.349)$ dos adolescentes avaliados apresentavam pelo menos uma lesão de cárie dentária; 55,57\% (n=1.336) tinham pelo menos um dente restaurado e 19,93\% $(\mathrm{n}=479)$ já haviam perdido um ou mais dentes permanentes. Na Tabela 2, verifica-se que o CPO -D médio foi de 3,69 $( \pm 3,26)$, representando, em média, $13,27 \%( \pm 11,68 \%)$ dos dentes avaliados. 
Tabela 2. Prevalência de dentes com experiência de cárie dentária em adolescentes. São Luís-MA, Brasil, 2016.

\begin{tabular}{lrrrrrrr}
\hline \multicolumn{1}{c}{ Variáveis } & $\mathbf{x}$ & $\mathbf{d p}$ & $\mathbf{Q 1}$ & Mediana & Q3 & Mín. & Máx. \\
\hline $\begin{array}{l}\text { Freq. Absoluta } \\
\quad \text { Cariado }\end{array}$ & 1,59 & 2,15 & 0,00 & 1,00 & 2,00 & 0,00 & 16,00 \\
$\quad$ Perdido & 0,34 & 0,82 & 0,00 & 0,00 & 0,00 & 0,00 & 8,00 \\
$\quad$ Obturado & 1,76 & 2,42 & 0,00 & 1,00 & 3,00 & 0,00 & 16,00 \\
$\quad$ CPO-D & 3,69 & 3,26 & 1,00 & 3,00 & 6,00 & 0,00 & 26,00 \\
Freq. relativa (\%) & & & & & & & \\
$\quad$ Cariado & 5,71 & 7,71 & 0,00 & 3,57 & 7,14 & 0,00 & 57,14 \\
$\quad$ Perdido & 1,23 & 2,94 & 0,00 & 0,00 & 0,00 & 0,00 & 28,57 \\
$\quad$ Obturado & 6,33 & 8,67 & 0,00 & 3,57 & 10,71 & 0,00 & 57,14 \\
$\quad$ CPO-D & 13,27 & 11,68 & 3,57 & 10,71 & 21,42 & 0,00 & 92,85 \\
\hline
\end{tabular}

x: média. Dp: desvio padrão. Q1: primeiro quartil. Q3: terceiro quartil. Max.: valor máximo. Mín.: valor mínimo.

Fonte: Elaborado pelas autoras.

$\mathrm{Na}$ análise não ajustada, associaram-se a maior prevalência de cárie não tratada os adolescentes que estavam casados ou morando com companheiro ( $\mathrm{RP}=1,33$; IC95\%: 1,17-1,51), residiam em casas com maior número de pessoas $(\mathrm{RP}=1,06$; IC95\%: 1,04-1,08), aqueles cujos pais estavam separados ( $\mathrm{RP}=1,13$; IC95\%: 1,05-1,21), que recebiam algum benefício social $(\mathrm{RP}=1,14$; IC95\%: 1,06-1,22), pertencentes às classes econômicas C (RP=1,26; IC95\%: 1,13-1,40) ou D-E $(\mathrm{RP}=1,56$; IC95\%: 1,41-1,74). Verificou-se menor ocorrência de cárie não tratada em adolescentes que estavam estudando por ocasião da pesquisa $(\mathrm{RP}=0,87$; IC95\%: 0,81-0,94) e que pertenciam a famílias com renda per capita três ou mais vezes acima da linha de pobreza ( $\mathrm{RP}=0,81$; IC95\%: 0,74-0,89).

Após ajuste do modelo, permaneceram associados a maior prevalência de cárie não tratada ser casado ou morar com companheiro ( $\mathrm{RP}=1,22$; IC95\%: 1,07-1,39), maior número de pessoas na residência $(\mathrm{RP}=1,05$; IC95\%: $1,03-1,07)$, ter pais separados ( $\mathrm{RP}=1,11$; IC95\%: 1,03-1,19) e pertencer às classes econômicas $C$ ( $\mathrm{RP}=1,23$; IC95\%:1,11-1,37) ou $\mathrm{D}-\mathrm{E}(\mathrm{RP}=1,48$; IC95\%: 1,32-1,65).

\section{Discussão}

Foram constatadas desigualdades injustas na distribuição da cárie em adolescentes de 18 a 19 anos do Município de São Luís-MA em relação aos fatores socioeconômicos. Pertencer às classes socioeconômicas C, D-E, estar casado, ser filho de pais separados e morar em residência com maior número de pessoas estiveram associados a maior prevalência de cárie dentária. Observa-se que a maior vulnerabilidade social pode influenciar na distribuição de condições bucais adversas em adolescentes, como a cárie dentária.

A prevalência de cárie não tratada entre os adolescentes da pesquisa foi de $56 \%$, resultado inferior ao encontrado no Brasil $(76,1 \%)$ e na Região Nordeste (77,1\%), segundo o último levantamento nacional de Saúde Bucal em 201027.

Já, se comparada à cidade de Goiânia $(54 \%)^{28}$ e à de Campina Grande $(38,5 \%)^{9}$, a prevalência de cárie encontrada em adolescentes foi maior. Segundo Silva et al.. ${ }^{29}$, as diferenças históricas no processo de ocupação e no desenvolvimento econômico das regiões brasileiras, bem como o desigual financiamento da saúde pública no início da implantação do Sistema Único de Saúde (SUS), no Brasil, justificam a desvantagem social das regiões Norte e Nordeste e, consequentemente, piores indicadores de saúde bucal.

O CPO-D médio encontrado $(3,69)$, considerado moderado ${ }^{26}$, mostrou-se inferior aos resultados do SB Brasil 2010 para a cidade de São Luís, que foi de $4,60^{27}$, e também inferior à região Nordeste no SB Brasil $2003(6,34)^{30}$. O componente "obturado" do índice CPO-D foi relevante $(1,76)$ em relação ao CPO-D total $(3,69)$ por expressar uma realidade de maior acesso por parte dos adolescentes avaliados.

Acredita-se que essa melhor condição registrada seja consequente de políticas públicas nacionais de saúde bucal que beneficiaram este e outros estratos etários nos últimos anos ${ }^{31-33}$. O último grande levantamento de cárie no Brasil foi realizado há quase 10 anos, portanto, pouco 
tempo após a implementação da Política Nacional de Saúde Bucal (PNSB).

Nossos dados, coletados em 2016, apontam para a redução da doença em uma população com grande vulnerabilidade socioeconômica. Entretanto, maiores prevalências de cárie foram identificadas nos segmentos socioeconômicos menos favorecidos. É provável que o acesso às medidas de promoção de saúde, prevenção e tratamento da doença seja também desigualmente distribuído ${ }^{34}$.

Os esforços da PNSB, com a criação de Centros de Especialidades Odontológicas (CEO), a inclusão, ainda que tardia, da equipe de saúde bucal (eSB) na Estratégia Saúde da Família (ESF) com foco na assistência longitudinal e familiar, e as alterações nas diretrizes curriculares do curso de Odontologia, com ênfase na formação de profissionais generalistas ${ }^{35-37}$, ainda não foram suficientes para eliminar as iniquidades em saúde bucal entre adolescentes, o que é evidenciado no presente estudo realizado em um dos estados mais pobres do Brasil.

Há necessidade não apenas de reduzir barreiras sócio-organizacionais e geográficas que possam tornar o acesso a serviços de saúde mais facilitado e homogêneo ${ }^{18}$. É ideal que haja investimento em capacitações para qualificação profissional, pois já foi demonstrado que é efetivo para ampliar a qualidade dos atributos da APS $^{38}$. Além do fortalecimento da Política Nacional de Educação Permanente em Saúde (PNEPS), na elaboração de estratégias com intuito de qualificar a atenção e gestão em saúde ${ }^{39}$.

O Pró-Saúde e o GraduaCEO foram iniciativas estabelecidas pelos Ministérios da Saúde e da Educação cujo objetivo era aproximar a teoria e prática lecionadas nas instituições de ensino com a realidade do SUS, como tentativa de modificar a formação em saúde ${ }^{40,41}$. Contudo, é necessário refletir acerca de um novo modelo assistencial voltado totalmente para a reorientação da formação, no qual se deve exigir comprometimento na atenção para o sujeito e determinantes sociais e atuação focada no SUS ${ }^{42}$. Isso pode ocorrer com a inserção de formandos em estágios no contexto da APS, como, por exemplo, participantes do Programa Saúde na Escola (PSE), com a disseminação de palestras sobre escovação dentária e uso do fio dental, aplicação de flúor e de selantes para evitar chegar a restaurações definitivas.

Este estudo provavelmente é um dos pioneiros a encontrar "estar casado ou morando com companheiro" como uma variável associada a maior prevalência de cárie em adolescentes
(22\%), visto não terem sido encontrados na literatura relatos de tal associação. Foi encontrada, em um estudo realizado com adultos, uma relação inversa da qual se afirma que adultos envolvidos em um relacionamento tendem a ter maior autocuidado e a existência de um cônjuge serve como um agente incentivador para o parceiro manter tratamentos de saúde ${ }^{11}$. Assim, acreditase que os adolescentes, diante das responsabilidades de uma vida adulta e conjugal, negligenciem os aspectos relativos à sua saúde. E é importante que doenças crônicas, como a cárie, sejam abordadas em adolescentes, pois existem chances de reversão de quadros considerados adversos que podem interferir de maneira negativa ao longo do ciclo da vida ${ }^{43}$.

Entre os que possuíam pais separados, a prevalência de cárie foi $11 \%$ maior do que entre os adolescentes com pais casados. Dados semelhantes foram encontrados no estudo de Ferrazano et al. ${ }^{44}$ e Pinto et al. ${ }^{45}$, nos quais a experiência de cárie entre adolescentes que não moravam com ambos os pais ou somente com as mães foi consideravelmente maior, podendo estar associada com a instabilidade emocional dos pais que, envolvidos em seus problemas, não priorizem a saúde bucal de seus filhos.

Os próprios adolescentes, diante da situação de estresse emocional e familiar, podem acabar modificando os hábitos, afetando sua saúde. A teoria do estresse ajuda a embasar essa hipótese. Segundo alguns autores ${ }^{6,15,46}$, um fator estressor pode prejudicar a saúde e o bem-estar psicológico do indivíduo que o enfrenta. A qualidade da saúde e a satisfação com a vida estão associadas às características sociais e econômicas dos indivíduos e ao meio ambiente em que residem, portanto, quanto mais estressante o ambiente, pior a qualidade de vida.

Em concordância com estudos anteriores ${ }^{47,48}$, morar em residências com maior número de pessoas também foi considerado um fator significantemente associado a maior prevalência de cárie dentária. Nessa situação, a superlotação no domicílio é um indicador de condição socioeconômica mais baixa, que por sua vez está associada a piores condições de saúde.

Após ajuste, o modelo revelou que pertencer às classes $\mathrm{C}$ e $\mathrm{D}-\mathrm{E}$, segundo a $\mathrm{ABEP}^{24,25}$, está intimamente relacionado à maior prevalência de cárie, sendo 23\% e 48\% maior, respectivamente, do que a prevalência encontrada em adolescentes da classe A-B. Porém, na Tabela 1, os resultados apontam uma divergência entre a classificação econômica e o indicador de renda $\mathrm{PIR}^{23}$. Ape- 
sar de a maioria da amostra pertencer à classe $\mathrm{C}$ (50,95\%), a renda predominante foi a considerada alta (PIR $\geq 3,0)$ entre $44,03 \%$ dos adolescentes. $\mathrm{Na}$ análise ajustada (Tabela 3 ) apenas a classe econômica associou-se à ocorrência de cárie. $\mathrm{O}$ fato revela que a variável "classe econômica" se mostrou mais sensível às variações do desfecho, o que pode ser explicado pelo fato de que ter uma renda familiar acima da média não implica necessariamente em melhorias socioeconômicas ou maior qualidade de vida ${ }^{49}$.

Boa parte da renda era proveniente de benefícios sociais, que podem ser interrompidos a cada mudança de governo ${ }^{50}$. Em consequência dessa instabilidade financeira, a renda pode não ser um bom indicador dos bens da família ${ }^{49}$. Há relação, no binômio mãe-filho, com a escolaridade materna e predição de cárie no filho e, assim, seria interessante considerar a escolaridade um indicador mais importante do que a renda em futuros estudos $^{51}$.

Diferentes mecanismos têm sido propostos para descrever os possíveis efeitos do status socioeconômico nos resultados de saúde. Uma possível explicação é que a debilidade dos laços de coesão social, ocasionada pelas iniquidades sociais, acarreta em escasso acesso à informação e ao conhecimento de aspectos fundamentais para promover boas condições de saúde, incluindo a bucal $^{52}$.

Tabela 3. Efeito das condições socioeconômicas na prevalência de cárie dentária não tratada em adolescentes. São Luís-MA, Brasil, 2016.

\begin{tabular}{|c|c|c|c|c|c|c|}
\hline \multirow{3}{*}{ Variáveis } & \multicolumn{6}{|c|}{ Prevalência de cárie dentária não tratada } \\
\hline & \multicolumn{3}{|c|}{ Não ajustada } & \multicolumn{3}{|c|}{ Ajustada } \\
\hline & $\mathbf{R P}$ & IC95\% & P-valor & RP & IC95\% & P-valor \\
\hline \multicolumn{7}{|l|}{ O adolescente está estudando atualmente? } \\
\hline Não & 1,00 & & & -- & -- & -- \\
\hline Sim & 0,87 & $0,81-0,94$ & $<0,001$ & -- & -- & -- \\
\hline \multicolumn{7}{|l|}{$\begin{array}{l}\text { O adolescente está trabalhando } \\
\text { atualmente? }\end{array}$} \\
\hline Não & 1,00 & & & -- & -- & -- \\
\hline Sim & 0,91 & $0,81-1,01$ & 0,081 & -- & -- & -- \\
\hline \multicolumn{7}{|l|}{ Situação conjugal do adolescente } \\
\hline Solteiro & 1,00 & & & 1,00 & & \\
\hline Casado/Morando com companheiro & 1,33 & $1,17-1,51$ & $<0,001$ & 1,22 & $1,07-1,39$ & 0,003 \\
\hline Número de pessoas na residência & 1,06 & $1,04-1,08$ & $<0,001$ & 1,05 & $1,03-1,07$ & $<0,001$ \\
\hline \multicolumn{7}{|l|}{ Os pais do adolescente estão separados } \\
\hline Não & 1,00 & & & 1,00 & & \\
\hline Sim & 1,13 & $1,05-1,21$ & 0,001 & 1,11 & $1,03-1,19$ & 0,004 \\
\hline \multicolumn{7}{|l|}{ Não informado } \\
\hline \multicolumn{7}{|l|}{$\begin{array}{l}\text { Alguém da residência recebe benefício } \\
\text { social? }\end{array}$} \\
\hline Não & 1,00 & & & & & \\
\hline Sim & 1,14 & $1,06-1,22$ & $<0,001$ & & & \\
\hline \multicolumn{7}{|l|}{ Classe econômica (ABEP) } \\
\hline \multicolumn{7}{|l|}{$A-B$} \\
\hline $\mathrm{C}$ & 1,26 & $1,13-1,40$ & $<0,001$ & 1,23 & $1,11-1,37$ & $<0,001$ \\
\hline $\mathrm{D}-\mathrm{E}$ & 1,56 & $1,41-1,74$ & $<0,001$ & 1,48 & $1,32-1,65$ & $<0,001$ \\
\hline \multicolumn{7}{|l|}{ Poverty income ratio $(\mathrm{PIR})^{1}$} \\
\hline Pobre $(\mathrm{PIR}<1,0)$ & 1,00 & & & 1,00 & & \\
\hline Quase pobre $(1,0 \leq \mathrm{PIR}<2,0)$ & 1,10 & $0,99-1,21$ & 0,062 & 1,07 & $0,97-1,18$ & 0,180 \\
\hline Renda mediana $(2,0 \leq \mathrm{PIR}<3,0)$ & 0,95 & $0,85-1,06$ & 0,381 & 0,97 & $0,87-1,08$ & 0,571 \\
\hline Renda alta (PIR $\geq 3,0)$ & 0,81 & $0,74-0,89$ & $<0,001$ & 0,92 & $0,83-1,01$ & 0,090 \\
\hline
\end{tabular}


Algumas limitações do estudo se referem à coleta de diferentes indicadores de condições socioeconômicas ao longo do ciclo da vida dos participantes. Isso impossibilitou a análise de mobilidade social de modo preciso, o que nos ajudaria a compreender o seu papel na prevalência de cárie dentária. Embora o estudo tenha utilizado dados de um seguimento da coorte RPS, foi o primeiro momento em que se realizaram exames clínicos odontológicos nos participantes, impossibilitando análises de incidência, apenas a prevalência da doença.

A relevância se deve à confirmação da persistência de iniquidades sociais, ainda diante de inúmeras ações implantadas há décadas e, também, à identificação de fatores individuais e coletivos de risco à cárie dentária, representados pelas condicionantes sociais, econômicos e culturais. O que permite corroborar a necessidade de qualificar a atenção à saúde bucal e a implementação de políticas públicas de promoção de saúde e prevenção de doenças, estruturadas por teorias contemporâneas e apropriadas para ações mais efetivas de forma que reduzam desigualdades.

Elementos de capital social tais como normas de harmonia ou solidariedade, confiança recíproca e engajamento cívico aliados ao crescimento da epidemiologia social, com foco na saúde do coletivo, são temas que poderão trazer novas perspectivas ao campo da saúde pública e da promoção de saúde. Não só por propor uma vida mais

\section{Colaboradores}

FS Sousa e BC Lopes contribuíram com o processo de escrita, análise dos dados e revisão final do artigo. EM Costa contribuiu com a coleta dos dados e revisão final do manuscrito. CMC Alves, RCS Queiroz, AS Tonello e CCC Ribeiro colaboraram no processo de concepção do estudo e revisão final do manuscrito. EBA Thomaz contribuiu no processo de concepção do estudo, análise estatística dos dados e revisão final do manuscrito. saudável para as populações a partir de mudanças comportamentais, como também para servir de meios para a possível redução de iniquidades sociais e com papel fundamental no estímulo à participação da comunidade tanto na formulação de políticas públicas, como no seu controle ${ }^{53,54}$.

\section{Conclusão}

A prevalência de cárie dentária em adolescentes na cidade de São Luís, no Maranhão, está associada a desvantagens socioeconômicas, especialmente ao número de pessoas na residência, à classe social, à situação conjugal dos seus pais e à do próprio adolescente. As iniquidades sociais em saúde bucal, ainda após grandes investimentos, persistem.

$\mathrm{O}$ conhecimento de fatores de risco à cárie dentária, representados pelos condicionantes sociais, econômicos e culturais, auxiliam na compreensão do processo saúde-doença em grupos sociais, bem como na identificação precoce de grupos de maior risco para receberem atenção preferencial em programas de saúde.

Assim, recomenda-se investir na qualificação da atenção à saúde bucal nas populações em situação de vulnerabilidade social e refletir sobre uma mudança no modelo assistencial para um que seja direcionado para a reorientação da formação.

\section{Financiamento}

As autoras são gratas às seguintes agências de pesquisa por seu apoio financeiro: Conselho Nacional de Desenvolvimento Científico e Tecnológico $(\mathrm{CNPq})$ e à Coordenação de Aperfeiçoamento de Pessoal de Nível Superior - Brasil (CAPES) - Código de Financiamento 001. 


\section{Referências}

1. Costacurta M, Epis M, Docimo R. Evaluation of DMFT in paediatric patients with social vulnerability conditions. Eur J Paediatr Dent 2020; 21(1):70-73.

2. Eid SA, Khattab NMA, Elheeny AAH. Untreated dental caries prevalence and impact on the quality of life among 11 to 14-year-old Egyptian schoolchildren: a cross-sectional study. BMC Oral Health 2020; 20(83):1-11.

3. Kastenbom L, Falsen A, Larsson P, SunnegårdhGrönberg K, Davidson T. Costs and health-related quality of life in relation to caries. BMC Oral Health 2019; 19(187):1-8.

4. Peres MA, Macpherson LMD, Weyant RJ, Daly B, Venturelli R, Mathur MR, et al. Oral diseases: a global public health challenge. Lancet 2019; 394(10219):249260.

5. Featherstone JDB. Dental caries: A dynamic disease process. Aust Dent J 2008; 53(3):286-291.

6. Folayan MO, Tantawi M El, Aly NM, Al-Batayneh OB, Schroth RJ, Castillo JL, Virtanen JI, Gaffar BO, Amalia R, Kemoli A, Vulkovic A, Feldens CA, the ECCAG. Association between early childhood caries and poverty in low and middle income countries. $\mathrm{BMC} \mathrm{Oral} \mathrm{He-}$ alth 2020; 20:8.

7. Mizuta A, Aida J, Nakamura M, Ojima T. Does the Association between Guardians' Sense of Coherence and their Children's Untreated Caries Differ According to Socioeconomic Status? Int J Environ Res Public Health 2020; 5(17):e1619.

8. Fonseca EP, Frias AC, Mialhe FL, Pereira AC, Meneghim MC. Factors associated with last dental visit or not to visit the dentist by Brazilian adolescents: A population-based study. PLoS One 2017; 12(8):e0183310.

9. Neves ÉTB, Dutra LC, Gomes MC, Paiva SM, Abreu MHNG, Ferreira FM, Granville-Garcia AF. The impact of oral health literacy and family cohesion on dental caries in early adolescence. Community Dent Oral Epidemiol 2020; 48(3):232-239.

10. Aguiar VR, Pattussi MP, Celeste RK. The role of municipal public policies in oral health socioeconomic inequalities in Brazil: A multilevel study. Community Dent Oral Epidemiol 2017; 46(3):245-250.

11. Lins LSS, Bezerra NVF, Freire AR, Almeida LFD, Lucena EHG, Cavalcanti YW. Socio-demographic characteristics are related to the advanced clinical stage of oral cancer. Med Oral Patol Oral y Cir Bucal 2019; 24(6):759-763.

12. Kramer A-CA, Petzold M, Hakeberg M, Östberg AL Multiple socioeconomic factors and dental caries in swedish children and adolescents. Caries Res 2018; 52(1-2):42-50.

13. Skeie MS, Klock KS. Dental caries prevention strategies among children and adolescents with immigrant-or low socioeconomic backgrounds-do they work? A systematic review. BMC Oral Health 2018; 18(1):20.

14. MacEntee MI, Wong ST, Chi I, Lo ECM, Minichiello V, Soheilipour S, Mariño R. Developmental regulation of lifelong dental experiences and beliefs in Guangzhou and Hong Kong. Gerodontology 2019; 36(1):1829.
15. Barauskas I, Barauskienė K, Janužis G. Dental anxiety and self-perceived stress in Lithuanian University of Health sciences hospital patients. A cross-sectional study. Stomatologija 2019; 21(2):42-46.

16. Vettore M V, Ahmad SFH, Machuca C, Fontanini H. Socio-economic status, social support, social network, dental status, and oral health reported outcomes in adolescents. Eur J Oral Sci 2019; 127(2):139-146.

17. Watt RG, Mathur MR, Aida J, Bönecker M, Venturelli R, Gansky SA. Oral Health Disparities in Children: A Canary in the Coalmine? Pediatr Clin North Am 2018; 65(5):965-979.

18. Guimarães RM. A teoria da equidade reversa se aplica na atenção primária à saúde? Evidências de 5.564 municípios brasileiros. Rev Panam Salud Publica 2018; 42:128.

19. Nickel DA, Lima FG, Silva BB. Modelos assistenciais em saúde bucal no Brasil. Cad Saude Publica 2008; 24(2):241-246.

20. Cardoso V, Barbieri M, Bettiol H, Goldani M, Silva A, Alves M. Profile of three Brazilian birth cohort studies in Ribeirão Preto, SP and São Luís, MA. Braz J Med Biol Res 2007; 40(9):1165-1176.

21. Moreira ARO, Batista RFL, Ladeira LLC, Thomaz EBAF, Alves CMC, Saraiva MC, Silva AAM, Brondani MA, Ribeiro CCC. Higher sugar intake is associated with periodontal disease in adolescents. Clin Oral Investig 2021; 25(3):983-991.

22. Harris PA, Taylor R, Thielke R, Payne J, Gonzalez N, Conde JG. Research Electronic Data Capture (REDCap) - A metadata-driven methodology and workflow process for providing translational research informatics support. J Biomed Inf 2009; 42(2):377-3381.

23. Capurro DA, Iafolla T, Kingman A, Chattopadhyay A, Garcia I. Trends in income-related inequality in untreated caries among children in the United States: Findings from NHANES I, NHANES III, and NHANES 1999-2004. Community Dent Oral Epidemiol 2015; 43(6):500-510.

24. Associação Brasileira de Empresas de Pesquisa (ABEP). Critério de Classificação Econômica do Brasil. São Paulo: ABEP; 2014.

25. Associação Brasileira de Empresas de Pesquisa (ABEP). Alterações na aplicação do Critério Brasil. São Paulo: ABEP; 2018.

26. World Health Organization (WHO). Oral health: Action plan for promotion and integrated disease prevention. In: WHO - Sixtieth World Health Assembly. Geneva: WHO; 2007.

27. Brasil. Ministério da Saúde (MS). Secretaria de Vigilância em Saúde. Secretaria de Atenção à Saúde. Coordenação Nacional de Saúde Bucal. SB Brasil 2010: Pesquisa Nacional de Saúde Bucal. Resultados Principais. Brasília: MS; 2012.

28. Oliveira LB, Moreira RS, Reis SCGB, Freire MDCM. Cárie dentária em escolares de 12 anos: Análise multinível dos fatores individuais e do ambiente escolar em Goiânia. Rev Bras Epidemiol 2015; 18(3):642-654.

29. Silva JV, Machado FCA, Ferreira MAF. Social inequalities and the oral health in Brazilian capitals. Cien Saude Colet 2015; 20(8):2539-2548. 
30. Brasil. Ministério da Saúde (MS). Secretaria de Vigilância em Saúde. Secretaria de Atenção à Saúde. Coordenação Nacional de Saúde Bucal. Projeto SB Brasil 2003: Condições de saúde bucal da população brasileira 2002-2003. Resultados Principais. Brasília: MS; 2004.

31. Bastos TF, Medina LPB, Sousa NFS, Lima MG, Malta DC, Barros MBA. Income inequalities in oral health and access to dental services in the Brazilian population: National health survey, 2013. Rev Bras Epidemiol 2019; 22(Supl. 2):e190015.

32. Castro RD, Rangel ML, Silva MAA, Lucena BTL, Cavalcanti AL, Bonan PRF, Oliveira JA. Accessibility to specialized public oral health services from the perspective of Brazilian users. Int J Environ Res Public Health 2016; 13:1026.

33. Hobdell M, Petersen PE, Clarkson J, Johnson N. Global goals for oral health 2020. Int Dent J 2003; 53(5):285-288.

34. Krupnikov Y, Levine AS. Political Issues, Evidence, and Citizen Engagement: The Case of Unequal Access to Affordable Health Care. Int J Polit 2019; 81(2):385398.

35. Brasil. Ministério da Saúde (MS). Secretaria de Atenção à Saúde. Departamento de Atenção Básica. Coordenação Nacional de Saúde Bucal. Diretrizes da Política Nacional de Saúde Bucal. Brasília: MS; 2004.

36. Brasil. Ministério da Educação (MEC). Diretrizes Curriculares Nacionais do Curso de Graduação em Odontologia. Brasília: MEC; 2002.

37. Brasil. Ministério da Saúde (MS). Secretaria de Atenção à Saúde. Departamento de Atenção Básica. Saúde Bucal. Brasília: MS; 2008.

38. Oliveira MPR, Menezes IHCF, Souza LM, Peixoto MRG. Formação e Qualificação de Profissionais de Saúde: Fatores associados à Qualidade da atenção Primária Training and Qualification of Health Professionals: Factors associated to the Quality of Primary Care. Rev Bras Educ Med 2016; 40(4):547-559.

39. Brasil. Ministério da Saúde (MS). Secretaria de Gestão do Trabalho e da Educação na Saúde. Departamento de Gestão da Educação na Saúde. Programa para o Fortalecimento das Práticas de Educação Permanente em Saúde no SUS (PRO EPS-SUS). Política Nacional de Educação Permanente em Saúde: o que se tem produzido para o seu fortalecimento? Brasília: MS; 2018.

40. Brasil. Ministério da Saúde (MS). Ministério da Educação (MEC). Portaria Interministerial no 3.019, de 26 de novembro de 2007. Dispõe sobre o Programa Nacional de Reorientação da Formação Profissional em Saúde - Pró-Saúde - para os cursos de graduação da área da saúde. Brasília; Diário Oficial da União 1990; 26 nov.

41. Brasil. Ministério da Saúde (MS). Portaria Interministerial $n^{\circ} 1.646$, de 5 de agosto de 2014. Institui o componente GraduaCEO - BRASIL SORRIDENTE, no âmbito da Política Nacional de Saúde Bucal, que irá compor a Rede de Atenção à Saúde (RAS), e dá outras providências. Brasília; Diário Oficial da União 1990; 5 ago.

42. Vendruscolo C, Trindade LDL, Prado ML, Kleba ME. Rethinking the Health Care Model through the reorientation of training. Rev Bras Enferm 2018; 71(Supl. 4):1580-1588.
43. Bezerra MRE, Lyra MJ, Santos MAM dos, Menezes VA. Fatores de Risco Modificáveis para Doenças Crônicas não Transmissíveis em Adolescentes: Revisão Integrativa. Adolesc Saude 2018; 15(2):113-120.

44. Ferrazzano GF, Sangianantoni G, Cantile T, Ingenito A. Relationship Between Social and Behavioural Factors and Caries Experience in Schoolchildren in Italy. Oral Health Prev Dent 2016; 14(1):55-61.

45. Pinto GDS, Hartwig AD, Elias R, Azevedo MS, Goettems ML, Correa MB, Demarco FF. Maternal care influence on children's caries prevalence in southern Brazil. Braz Oral Res 2016; 30(1):S180683242016000100262.

46. Akinkugbe AA, Hood KB, Brickhouse TH. Exposure to Adverse Childhood Experiences and Oral Health Measures in Adulthood: Findings from the 2010 Behavioral Risk Factor Surveillance System. JDR Clin Trans Res 2019; 4(2):116-125.

47. Cable N, Sacker A. Validating overcrowding measures using the UK Household Longitudinal Study. SSM Popul Health 2019; 8:100439.

48. Russell J, Grant CC, Morton SMB. Multimorbidity in Early Childhood and Socioeconomic Disadvantage: Findings From a Large New Zealand Child Cohort. Acad Pediatr 2020; 20(5):619-627.

49. Browne-Yung K, Ziersch A, Baum F. "Faking til you make it": Social capital accumulation of individuals on low incomes living in contrasting socio-economic neighbourhoods and its implications for health and wellbeing. Soc Sci Med 2013; 85:9-17.

50. Calvasina P, O'Campo P, Pontes MM, Oliveira JB, Vieira-Meyer APGF. The association of the Bolsa Familia Program with children's oral health in Brazil. BMC Public Health 2018; 18(1186):1-10.

51. Silva MGB, Catão MHCV, Andrade FJP, Alencar CRB. Cárie precoce da infância: fatores de risco associados. Arch Health Investig 2017; 6(12):574-579.

52. Barata RB. O que queremos dizer com desigualdades sociais em saúde? In: Rita BB, organizadora. Como e por que as desigualdades sociais fazem mal à saúde. Rio de Janeiro: Fiocruz; 2009. p. 11-22.

53. Campbell C. Social capital, social movements and global public health: Fighting for health-enabling contexts in marginalised settings. Soc Sci Med 2019; 257:112153.

54. Wind TR, Villalonga-Olives E. Social capital interventions in public health: Moving towards why social capital matters for health. $J$ Epidemiol Community Health 2019; 73(9):793-795.

Artigo apresentado em 22/05/2020

Aprovado em 14/04/2021

Versão final apresentada em 16/04/2021

Editores-chefes: Romeu Gomes, Antônio Augusto Moura da Silva 